\title{
Salt and Pepper Noise Removal Filter for 8-Bit Images Based on Local and Global Occurrences of Grey Levels as Selection Indicator
}

\author{
Isma Irum, Muhammad Sharif, Mudassar Raza, Mussarat Yasmin \\ COMSATS Institute of Information Technology, Wah Cantt, Pakistan \\ e-mail: mussaratyasmin@comsats.edu.pk
}

\begin{abstract}
A spatial resolution decision based trimmed moving window filtering method has been proposed for salt and pepper noise removal. It provides a criterion for the selection of neighboring pixels based on the probabilities of local and global occurrences of grey levels of noisy image. It works well for the high noise densities up to $90 \%$ and contributes very effectively in estimating the true or near to true values of the original image. In order to measure the quality of image, well known quantification measures SSIM and PSNR are used. The method is compared with other existing techniques and shows that it outperforms other methods in terms of PSNR.
\end{abstract}

Key words: Decision based filtration, image de noising, image restoration, impulse noise, trimmed moving window

\section{Introduction}

Bit errors created during image transmission and acquisition lead to creation of impulse noise. There exists impulse noise of two types: one is called random valued impulse noise and the other one is categorized as salt and pepper noise. Only two grey level values; maximum or minimum can be taken by the salt and pepper noise. For images that have been contaminated by salt and pepper noise, many nonlinear filters have been proposed for the restoration of image. Among these non linear filters, standard median filter has been proved to be very effective to preserve the edge details after the removal of salt and pepper noise. However in case of high noise densities, standard median filter (MF) does not effectively eliminate the salt and pepper noise as described by Astola \& Kuosmaneen (1997). Up to $50 \%$ of noise levels standard median filter can preserve the edges detail. For densities of low noise, adaptive median filter proposed by Hwang and Hadded (1995) is quite effective but the window size increment results in image blurring as far as densities of high noise is concerned.

Among the category of decision based methods, switching median filters (Ng \& Ma 2006, Zhang \&
Karim 2002) are dependent on a predefined value of threshold but mainly its disadvantage is designing of a robust decision which is very difficult to do. Because these filters do not take into consideration the local features, therefore, the details of image are not satisfactorily preserved regarding noise of high density.

Decision based algorithm (DBA) (Srinivasan \& Ebenezer 2007) has been presented to solve this problem. It uses a window of $3 \times 3$ sizes. It gets processed if the considered pixel value is 0 or 255 otherwise it is not changed. When the density of noise is high the neighboring pixels constitute the values of 0 and 255 hence yielding the median values 0 and 255 which is noise. In this case the repeated selection of neighboring pixels produce streaking effect (Jayaraj \& Ebenezer 2010). Decision Based Un-symmetric Trimmed Median Filter (DBUTM) (Aiswarya et al. 2010) has been proposed to handle this limitation. This algorithm also creates problem of finding trimmed median filter in case of high densities when all the neighboring pixels constitute the values 0 and 255 .

The proposed filter provides a flexible criterion for the selection of neighboring pixels even in case of high 
noise densities using the local and global occurrences of grey levels. If pixel under processing is 0 or 255 it is processed otherwise left unchanged. It excludes the noisy pixels existing in neighboring pixels of moving window. The neighboring pixel selected for the replacement of centered noisy pixel is based on the neighboring pixel that contains the maximum sum of probabilities of local and global occurrence. As compared to the presented algorithms of filtration, better values of SSIM and PSNR are obtained by it.

The paper is written in the sequence: proposed work is presented in section 2 and image fidelity measures that are used to quantify the image are discussed in section 3. Section 4 includes the results of experiments as well as a discussion on these results.

\section{Methodology}

The proposed method is categorized as spatial resolution decision based trimmed moving window filtering technique. It utilizes the sum of probabilities of local occurrences of grey levels in moving window and global occurrences of grey levels in the whole image. A constant window size of $3 \times 3$ is taken to save the execution time and efficiency of method. Local and global grey levels help in restoring the noisy image by selecting the existing grey levels in the whole image as a selection indicator of selectable value for noisy pixel replacement. It preserves the image smoothness, details and integrity.

\section{Noise Model}

Many kinds of noise can exist in images as a result of applying different procedures on them like compression, data transmission and acquisition etc. Among all these types of noise, impulse noise is categorized as the most popular and common one. This noise actually changes the pixels intensities of an image up to some maximum or minimum range of intensities. Noise model used in the proposed approach is as described in (Junez-Ferreira et al.ss ). Let $\mathrm{x}_{\mathrm{i}, \mathrm{j}}$, for $(\mathrm{i}, \mathrm{j}) \square \mathrm{A}$, where A $\{1 \ldots \ldots \ldots \mathrm{R}\} \times\{1 \ldots \ldots \ldots \mathrm{C}\}$, the intensity value of a pixel of a $\mathrm{R} \times \mathrm{C}$ sized image $\mathrm{x}$ at position $(i, j)$ and let $\left[\mathrm{v}_{\min }, \mathrm{v}_{\max }\right]$ the dynamic range of $\mathrm{x}$, i.e., noise corrupted version of $\mathrm{x}$, then the observed intensity value at position $(i, j)$ of image $y$ is given by

$$
y_{i, j}=\left\{\begin{array}{l}
v_{\min } \text { with probability } a \\
v_{\max } \text { with probability } b \\
x_{i, j} \text { with probability } 1-a-b
\end{array}\right\}
$$

Where $\mathrm{r}=\mathrm{a}+\mathrm{b}$ defines noise density. In this work, it is considered that $a=b$.

Removal of noise from an image is important because of the reason of getting back the approximate original image after applying the noise suppression process on it.

\section{Noise Detection}

The traditional noise detection approaches used to apply the noise detection process unconditionally on all the pixels without taking into consideration that the pixels are corrupted or not. The result is that the quality of image gets affected and the uncorrupted pixels still need to be filtered out. The solution is to apply some noise detection process before starting the filtration process so that only those pixels pass through the process of filtration which is targeted as uncorrupted pixels.

The noise detection process presented in the paper is very simple as shown in Figure 1. By moving the working window pixel by pixel on all spatial resolution of image in the Binary Map, each value is observed. If it is either equal to the maximum intensity or equal to the minimum intensity as defined by the noise model then it is marked as noisy pixel (np) and indicated by ' 0 ' in binary map. Uncorrupted pixels are indicated by ' 1 ' in binary map. In this case the maximum and minimum intensities are defined to be ' 255 ' and ' 0 ' respectively. $n p$ can be defined by the equation

$$
f\left(y_{i, j}\right)=\{n p \backslash y(i, j)=0,255\}
$$

\section{Moving Window}

Moving window is a mask structure of $3 \times 3$ but the size used in the proposed approach is selected by summing up the values of binary map. Moving window size (mws) is given by the following equation

$$
m w s=\sum_{p=1}^{3} \sum_{q=1}^{3} u_{p, q}
$$

Where p represents rows and q represents columns in the Binary Map. $\mu_{p, q}$ is an indication of a value in the Binary Map at some row and column.

\section{Probability of Grey Levels (PG)}

In an image of 8-bit, there exist a total of $2^{8}=256$ grey scale levels from 0 to 255 . An image density 
probability distribution of the pixels values over the entire grey scale range is represented by an image histogram.

Let $x$ be a grey scale image and the grey level $i$ number of occurrences be represented by $n_{i}$. Then the level $i$ pixel occurrence probability in an image is given by: $P_{(x)} i=n_{i} / n$ such that $0 \leq i<L$

Where an image's total pixels is represented by $n$ and an image's total number of grey levels is represented by $L$.

\section{Local Probability of Grey Levels (LPG)}

Local probability of grey levels (LPG) is defined as the probability of occurrences of grey levels in moving window. It can be obtained by dividing the value of spatial resolution of pixel by size of moving window. It can be given by the following equation

$$
\operatorname{LPG}\left(u_{p, q}\right)=\frac{u_{p, q}}{m w s}
$$

Window Slide Direction

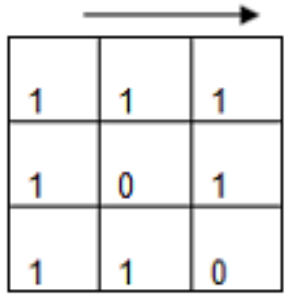

a. Binary Map

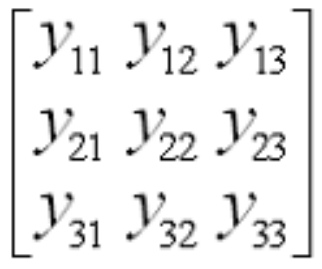

b. Moving Window

Fig. Moving Window and Binary Map

\section{Global Probability of Grey Levels (GPG)}

Global probability of grey levels (GPG) is defined as the probability of occurrences of grey levels in the whole image. For an 8-bit image grey level has dynamic range [0-255] but here ' 0 ' and '255' are excluded as indicated nps by noise detection. GPG is given by the following equation

$$
G P G=\frac{1}{R C} \sum x(a) \text { for } a=1 \ldots 254
$$

\section{Filtering Scheme}

Among the many existing filtering schemes for the removal of impulse noise, the most commonly used one is termed as median filter. Although this filter is quite effective in its working but it performs modification on both the noise free as well as noisy pixels as a result of which some necessary details also get removed from the image.

In order to overcome this limitation of median filter, many other filtering schemes have been proposed like pixel wise MAD filter, tri-state median filter and center-weighted median filter etc. Performance evaluation of all these introduced filters is dependent on the detector used in these filters. Moreover, most of the filters do not remain efficient in case the impulse noise has random values.

As it is already mentioned that the proposed method is characterized as spatial resolution decision based trimmed moving window filtering technique, therefore a value replaces the moving window central pixel. This value is decided by a filtering scheme that is discussed here. The steps given below describe the filtering scheme.

1. Calculate the sum of LPG and its corresponding GPG for $\mathrm{u}_{\mathrm{pq}}$ existing in moving window, it is called selection indicator (SI) as shown in Figure 2 and given by

$$
S I\left(u_{p, q}\right)=L P G\left(u_{p, q}\right)+G P G\left(u_{p, q}\right) \text { for } 1 \leq p, q \leq 3
$$

$$
\left[\begin{array}{lll}
x_{11} & x_{12} & x_{13} \\
x_{21} & x_{22} & x_{23} \\
x_{31} & x_{32} & x_{33}
\end{array}\right]
$$

$$
\left[\begin{array}{lll}
S I_{11} & S I_{22} & S I_{33} \\
S I_{21} & 0 & S I_{23} \\
S I_{31} & S I_{32} & S I_{33}
\end{array}\right]
$$

\section{b. Corresponding SI}

Fig. Moving Window with Its Selection Indicator

2. Find the maximum of 'SI' and index of maximum is returned.

$$
\text { ind }[p, q]=\operatorname{ind}[\max (S I)]
$$

3. Finally, the corresponding upq is selected to replace the $\mathrm{np}$. 


$$
n p=y_{\text {ind }[p, q]}
$$

The above three steps are repeated until all the nps are replaced by neighborhood value selected by the above filtering scheme.

\section{Image Fidelity Measures}

With reference to an original image, different quality measures are to be calculated for a noisy image. These measures include MSE (Mean Square Error), PSNR (Peak Signal to noise Ratio in dB), MD (Maximum Difference), AD (Average Difference), NAE (Normalized Absolute Error) and NCC (Normalized Cross Correlation) etc.

Selection of appropriate fidelity measures is a challenging task depending upon a number of criteria including guidance of parameter selection, designing of objective function and making decisions etc.

PSNR (dB), execution time and SSIM (Wang et al. 2004) are used to quantify the quality of image restored by the proposed method.

SSIM index is an improvement on the methods in which inconsistencies occur because of human eye perception. It is probably the most effective quality measure giving quite reliable and comfortable results. Task of similarity measurement in exploring structural information of an image is divided into comparisons of three types.

$$
\begin{array}{ll}
\circ & \text { structure } \mathrm{s}(\mathrm{x}, \mathrm{y}) \\
\circ & \text { luminance } \mathrm{l}(\mathrm{x}, \mathrm{y}) \text {; } \\
\circ & \text { contrast } \mathrm{c}(\mathrm{x}, \mathrm{y}) ;
\end{array}
$$

SSIM gives the equation as follows (Wang, et al. 2004)

$$
\operatorname{SSMM}(x, y)=[l(x, y)]^{\alpha}[c(x, y)\}^{\beta}[s(x, y)]^{y}(9)
$$

Where equation 10 to equation 12 give $s(x, y), 1(x, y)$ and $\mathrm{c}(\mathrm{x}, \mathrm{y})$ respectively and the components importance is adjusted by the $\alpha>0, \beta>0, \psi 0$ parameters.

$$
l(x, y)=\frac{2 \mu_{x} \mu_{y}+C_{1}}{\mu_{x}^{2}+\mu_{y}^{2}+C_{1}}
$$

Where constant $C_{1}$ has the value ${ }^{\mathrm{D}} K_{1} L^{\mathrm{C}_{2}}$ for $K_{1} \ll<1$ and mean intensities of original and restored images respectively are denoted by $\mu_{y}, \mu_{x}$.

$$
c(x, y)=\frac{2 \sigma_{x} \sigma_{y}+C_{2}}{\sigma_{x}^{2}+\sigma_{y}^{2}+C_{2}}
$$

Where constant $C_{2}$ has the value ${ }^{\mathrm{b}} K_{2} L^{\mathrm{C}_{2}}$ for $K_{2} \ll<1$ and original and restored images intensities have $\sigma_{y}, \sigma_{x}$ as the standard deviations respectively.

$s(x, y)=\frac{\sigma_{x y}+C_{3}}{\sigma_{x}+\sigma_{y}+C_{3}}$

Where constant is denoted by $C_{3}$ and between original and restored image, the cross correlation is denoted by $\sigma_{x y}$. Parameters are used with the v a 1 u e s $\alpha=\beta=\gamma=1, K_{1}=001, K_{2}=003 C_{3}=\frac{f}{2}$ according to [SSIM] equation. Computation of local window SSIM is done with a $3 \times 3$ sized window throughout the pixel by pixel.

PSNR is the most used image quality measure in which noise is the error given by the process of image compression. It is an approximation to the reconstruction quality of what the human perceives. Although the higher PSNR value indicates the reconstruction of high quality but it is not the case in some situations. So, one has to be careful regarding range validation of this fidelity measure.

PSNR can be given by the following equation

$$
P S N R=10 \log _{10}\left(\frac{L^{2}}{M S E}\right)
$$

In this case, $\mathrm{L}=255$ (maximum dynamic range) for 8bit images.

MSE measures the average of the square of errors i.e., it computes the difference between what is estimated and the estimator. In short, MSE is the variance estimator. It bears the same measurement units as the square of estimated quantity.

MSE is given by

$$
M S E=\frac{1}{R C} \sum_{=}^{g} \sum_{=}^{C}\left(\mathrm{Y}_{\mathrm{i}, \mathrm{j}}-\mathrm{X}_{\mathrm{i}, \mathrm{j}}\right)^{2}
$$

Where $y_{i, j}$ is the pixel intensity of original image at location ( $\mathrm{i}, \mathrm{j})$, and $x_{i, j}$ is the pixel intensity of restored image at location $(i, j)$. 


\section{Results and Discussion}

The proposed method has been applied on various standard 8-bit monochromatic images used in (Weber 1997). All images are of size 512x512. Experimental results of six images are discussed and shown here as given in Figure 3.

Table 1 gives the PSNR of noisy images and restored images. Table 2 shows the execution time of proposed
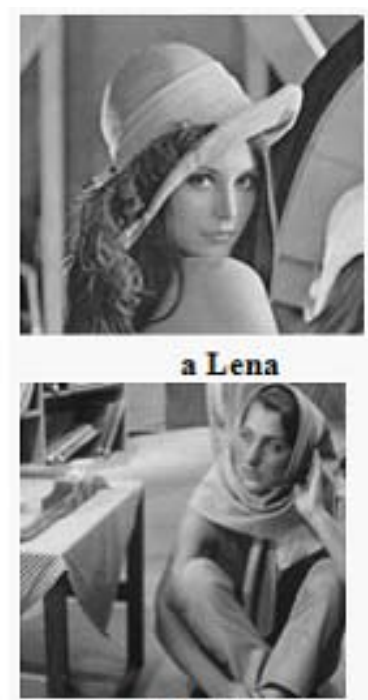

d Barbara

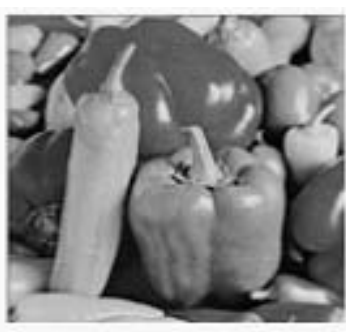

B Peppers

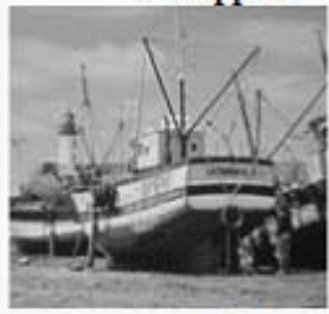

e Boat method. SSIM of noisy and restored images is given in Table 3. Table 4 presents the PSNR (dB) comparison of proposed filter with other existing algorithms (Esakkirajan et al. 2011) where proposed filter values are appended in bold face in last column of the table. Figure 4 shows the comparison of PSNR (dB) for proposed filter with other existing techniques whereas noisy images and restored images are shown in Figure 5.

Fig. 3. Original Images

Table1. shows that there is almost an increase of $15(\mathrm{~dB})$ to $22(\mathrm{~dB})$ of PSNR improvement from noisy image to restored image.

Table 1. PSNR(dB) of noisy and restored images

\begin{tabular}{|c|c|c|c|c|c|c|c|c|c|c|}
\hline $\begin{array}{c}\text { Noise } \\
\text { Density }\end{array}$ & \multicolumn{2}{|c|}{$10 \%$} & \multicolumn{2}{|c|}{$20 \%$} & \multicolumn{2}{|c|}{$30 \%$} & \multicolumn{2}{|c|}{$40 \%$} & \multicolumn{2}{|c|}{$50 \%$} \\
\hline Images & $\begin{array}{l}\text { Noisy } \\
\text { Imag }\end{array}$ & $\begin{array}{l}\text { Restored } \\
\text { Image }\end{array}$ & $\begin{array}{l}\text { Noisy } \\
\text { Image }\end{array}$ & $\begin{array}{l}\text { Restored } \\
\text { Image }\end{array}$ & $\begin{array}{l}\text { Noisy } \\
\text { Image }\end{array}$ & $\begin{array}{l}\text { Restored } \\
\text { Image }\end{array}$ & $\begin{array}{l}\text { Noisy } \\
\text { Image }\end{array}$ & $\begin{array}{l}\text { Restored } \\
\text { Image }\end{array}$ & $\begin{array}{l}\text { Noisy } \\
\text { Image }\end{array}$ & $\begin{array}{l}\text { Restored } \\
\text { Image }\end{array}$ \\
\hline Lena & 37.11 & 57.25 & 34.05 & 54.79 & 32.28 & 53.49 & 31.08 & 53.48 & 30.10 & 52.57 \\
\hline Peppers & 37.14 & 57.97 & 34.12 & 55.42 & 32.37 & 54.21 & 31.08 & 54.26 & 30.12 & 52.58 \\
\hline Baboon & 37.05 & 52.14 & 34.09 & 49.08 & 32.33 & 48.04 & 31.06 & 47.68 & 30.08 & 45.51 \\
\hline Barbara & 37.05 & 54.92 & 34.08 & 52.28 & 32.32 & 51.46 & 31.06 & 50.53 & 30.09 & 48.85 \\
\hline Boat & 37.17 & 56.69 & 34.06 & 53.96 & 32.32 & 52.86 & 31.06 & 50.50 & 30.09 & 49.55 \\
\hline Gold hill & 37.00 & 56.67 & 34.06 & 54.18 & 32.30 & 52.26 & 31.08 & 50.35 & 30.09 & 48.17 \\
\hline $\begin{array}{l}\text { Noise } \\
\text { Density }\end{array}$ & & $60 \%$ & & $0 \%$ & & $0 \%$ & & $\% \%$ & & \\
\hline Lena & 29.29 & 50.13 & 28.63 & 49.49 & 28.06 & 48.99 & 27.52 & 46.27 & & \\
\hline Peppers & 29.32 & 50.73 & 28.68 & 48.77 & 28.09 & 48.15 & 27.58 & 46.04 & & \\
\hline Baboon & 29.31 & 42.89 & 28.62 & 41.39 & 28.06 & 38.94 & 27.53 & 36.63 & & \\
\hline Barbara & 29.28 & 46.20 & 28.61 & 46.77 & 28.62 & 44.87 & 27.53 & 44.11 & & \\
\hline Boat & 29.30 & 48.16 & 28.62 & 46.69 & 28.04 & 44.78 & 27.52 & 44.10 & & \\
\hline
\end{tabular}


Nepal Journal of Science and Technology Vol. 15, No.2 (2014) 123-132

Table 2. Execution time (sec)

\begin{tabular}{c|c|c|c|c|c|c|c|c|c}
\hline Noise Density & $10 \%$ & $20 \%$ & $30 \%$ & $40 \%$ & $50 \%$ & $60 \%$ & $70 \%$ & $80 \%$ & $90 \%$ \\
\hline Lena & 1.31 & 1.65 & 2.03 & 2.43 & 2.95 & 3.16 & 3.56 & 3.89 & 4.29 \\
Peppers & 1.26 & 1.60 & 2.03 & 2.54 & 2.87 & 3.24 & 3.62 & 3.96 & 4.43 \\
Baboon & 1.26 & 1.61 & 1.99 & 2.35 & 2.73 & 3.12 & 3.48 & 3.84 & 4.21 \\
Barbara & 1.63 & 1.59 & 2.04 & 2.37 & 2.74 & 3.12 & 3.49 & 3.85 & 4.18 \\
Boat & 1.35 & 1.67 & 2.04 & 2.35 & 2.71 & 3.08 & 3.46 & 3.82 & 4.28 \\
Goldhill & 1.15 & 1.62 & 1.96 & 2.32 & 2.76 & 3.16 & 3.57 & 3.90 & 4.18 \\
\hline
\end{tabular}

Table 3. SSIM values of noisy and restored images

\begin{tabular}{|c|c|c|c|c|c|c|c|c|c|c|}
\hline \multirow[t]{2}{*}{$\begin{array}{l}\text { Noise } \\
\text { Density }\end{array}$} & \multicolumn{2}{|l|}{$10 \%$} & \multicolumn{2}{|l|}{$20 \%$} & \multicolumn{2}{|l|}{$30 \%$} & \multicolumn{2}{|l|}{$40 \%$} & \multicolumn{2}{|l|}{$50 \%$} \\
\hline & Noisy & Restored & Noisy & Restored & Noisy & Restored & Noisy & Restored & Noisy & Restored \\
\hline Images & Image & Image & Image & Image & Image & Image & Image & Image & Image & Image \\
\hline Lena & 0.46 & 0.99 & 0.32 & 0.97 & 0.24 & 0.94 & 0.18 & 0.84 & 0.15 & 0.49 \\
\hline Peppers & 0.46 & 0.99 & 0.33 & 0.98 & 0.25 & 0.94 & 0.19 & 0.82 & 0.14 & 0.41 \\
\hline Baboon & 0.60 & 0.96 & 0.44 & 0.92 & 0.33 & 0.85 & 0.25 & 0.72 & 0.18 & 0.48 \\
\hline Barbara & 0.55 & 0.98 & 0.39 & 0.94 & 0.31 & 0.82 & 0.23 & 0.78 & 0.18 & 0.41 \\
\hline Boat & 0.47 & 0.99 & 0.35 & 0.97 & 0.27 & 0.92 & 0.21 & 0.83 & 0.16 & 0.59 \\
\hline Goldhill & 0.49 & 0.99 & 0.33 & 0.96 & 0.24 & 0.91 & 0.18 & 0.77 & 0.14 & 0.32 \\
\hline $\begin{array}{l}\text { Noise } \\
\text { Density }\end{array}$ & $60 \%$ & & $70 \%$ & & $80 \%$ & & $90 \%$ & & & \\
\hline Lena & 0.10 & 0.33 & 0.08 & 0.28 & 0.05 & 0.24 & 0.03 & 0.23 & & \\
\hline Peppers & 0.12 & 0.28 & 0.08 & 0.25 & 0.06 & 0.22 & 0.03 & 0.20 & & \\
\hline Baboon & 0.13 & 0.36 & 0.09 & 0.30 & 0.06 & 0.26 & 0.04 & 0.22 & & \\
\hline Barbara & 0.13 & 0.29 & 0.09 & 0.24 & 0.06 & 0.21 & 0.04 & 0.17 & & \\
\hline Boat & 0.12 & 0.48 & 0.09 & 0.42 & 0.06 & 0.36 & 0.04 & 0.33 & & \\
\hline Goldhill & 0.10 & 0.22 & 0.07 & 0.19 & 0.05 & 0.18 & 0.03 & 0.16 & & \\
\hline
\end{tabular}

As described in (Wang, et al. 2004) the value of SSIM values near to 1 indicate good image quality. It can be varies between [0-1], the ideal case is 1 . However seen from Table 3 that all resulting outcomes are higher values.

Table 4. Different algorithms PSNR comparison at different noise densities for image of Lena

\begin{tabular}{l|l|l|l|l|l|l|l}
\hline & \multicolumn{7}{|c}{ PSNR in dB } \\
\hline Noise Density & MF & AMF & PSMF & DBA & MDBA & MDBUTMF & Proposed Filter \\
$10 \%$ & 26.34 & 28.43 & 30.22 & 36.40 & 36.94 & 37.91 & 57.25 \\
$20 \%$ & 25.66 & 27.40 & 28.39 & 32.90 & 32.69 & 34.78 & 54.79 \\
$30 \%$ & 21.86 & 26.11 & 25.52 & 30.15 & 30.41 & 32.29 & 53.49 \\
$40 \%$ & 18.21 & 24.40 & 22.49 & 28.49 & 28.49 & 30.32 & 53.48 \\
$50 \%$ & 15.04 & 23.36 & 19.13 & 26.41 & 26.52 & 28.18 & 52.57 \\
$60 \%$ & 11.08 & 20.60 & 12.10 & 24.83 & 24.41 & 26.43 & 50.13 \\
$70 \%$ & 9.93 & 15.25 & 9.84 & 22.64 & 22.47 & 24.30 & 49.49 \\
$80 \%$ & 8.68 & 10.31 & 8.02 & 20.32 & 20.44 & 21.70 & 48.99 \\
$90 \%$ & 6.65 & 7.93 & 6.57 & 17.14 & 17.56 & $18.40[12]$ & 46.27 \\
\hline
\end{tabular}


Isma Irum et al/Salt and Pepper Noise......
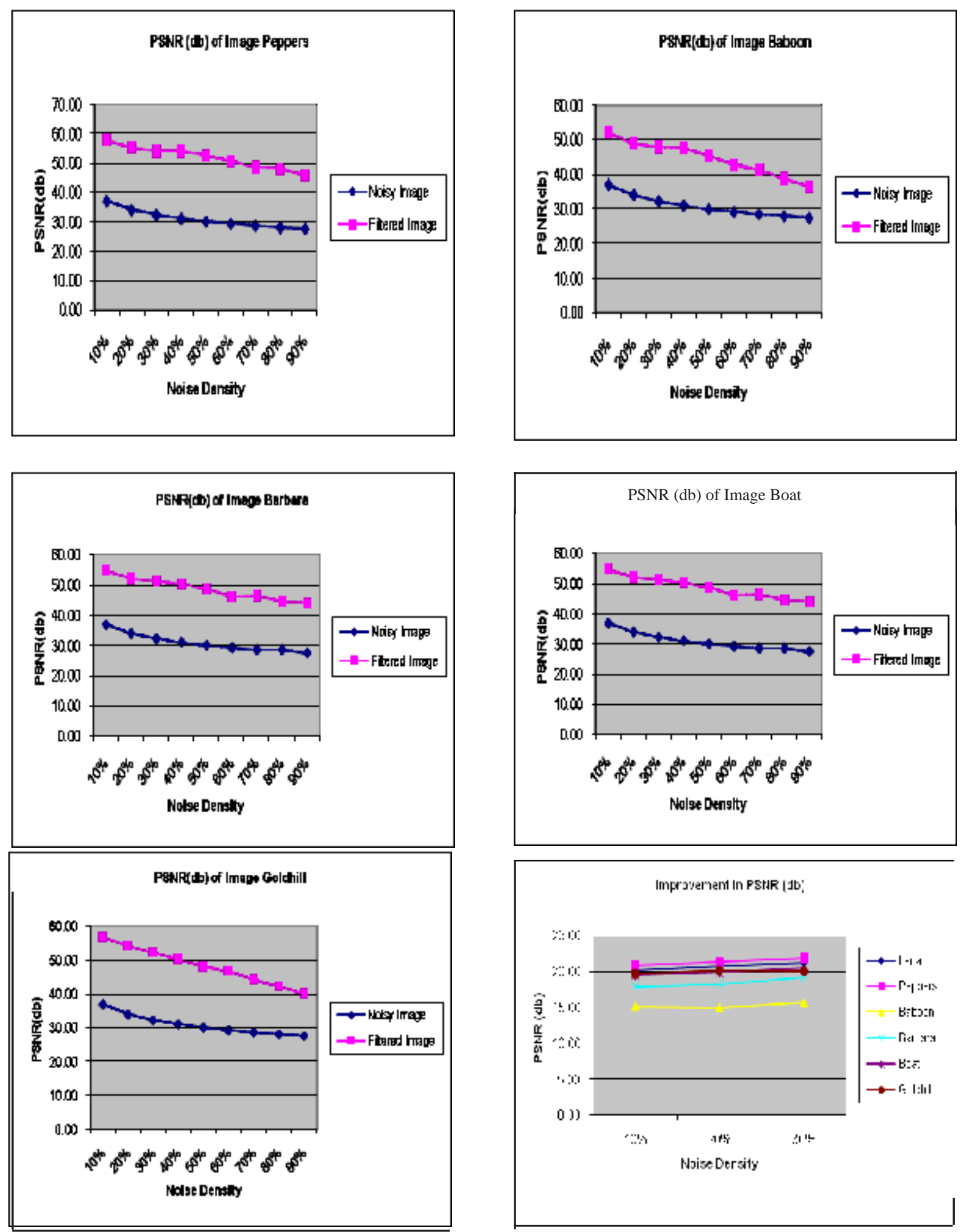

Fig.4. PSNR (dB) Comparison: Proposed filter and existing techniques 


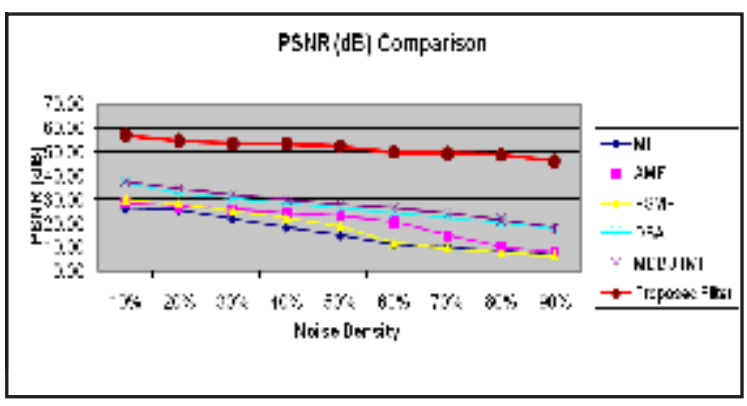

Fig.4. PSNR (dB) Comparison: Proposed filter and existing techniques

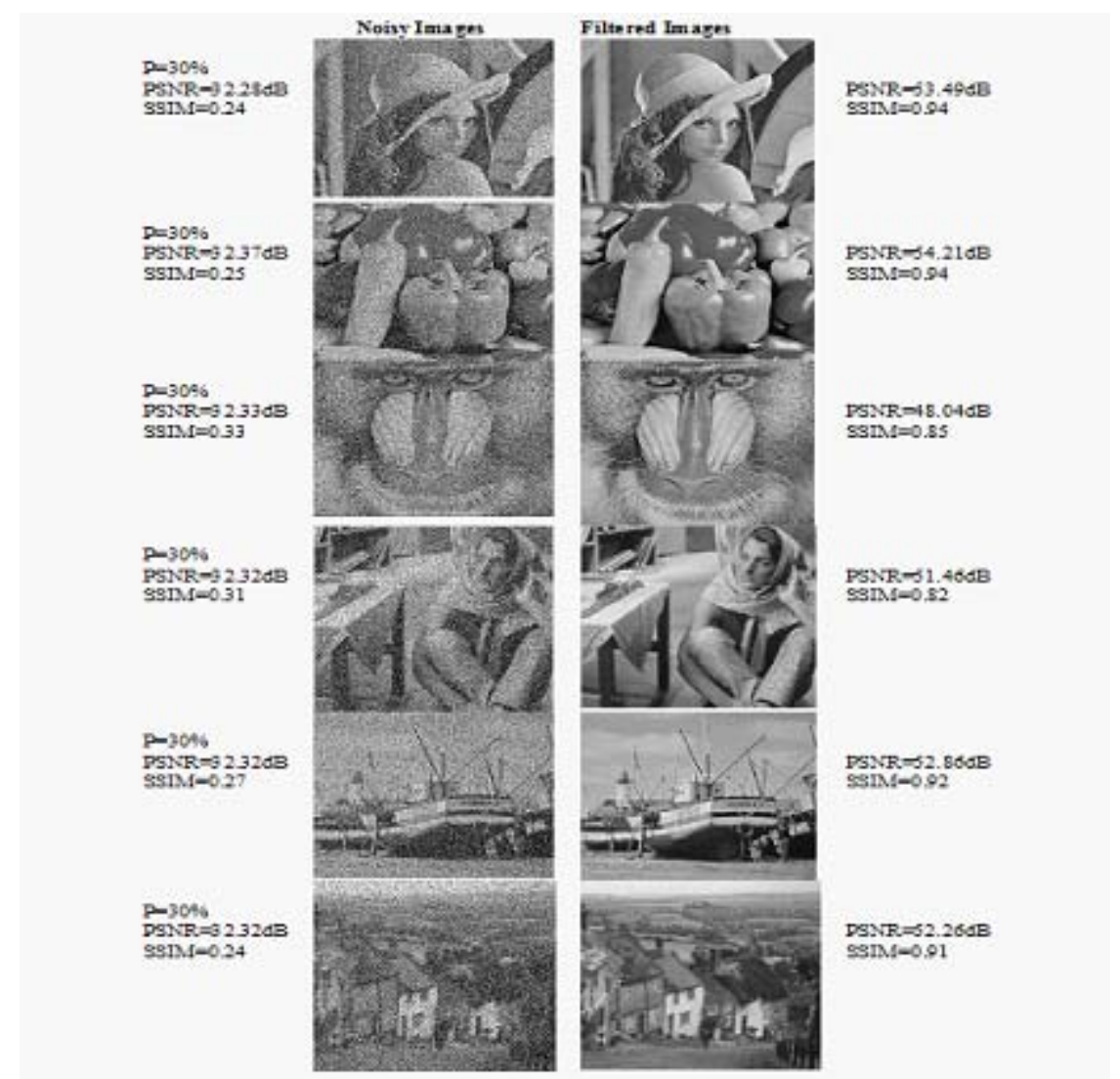

Fig. 5. Restored image results of proposed method for images Lena, Peppers, Baboon, Barbara, Boat and Goldhill

An efficient salt and pepper noise removal method has been proposed that removes the noise up to $90 \%$ of density and secures very high PSNR and SSIM values. The proposed approach selects those values of neighborhood to replace the noisy pixels which have more occurrences in the whole image. In this way it distributes the normalized values of grey levels while restoring the image. Image quality obtained is very good and image details and edges integrity are also preserved.
Proposed method outperforms in terms of PSNR, SSIM and execution time as from Figure 5 of noisy and restored images it can be seen that the restored image quality is visually very good and image details and edges integrity are preserved. It can also be observed from Figure 4 that the proposed method achieves the highest values for PSNR as compared to existing techniques. Table 4 also clearly shows the highest achieved PSNR values of proposed filter that strengthens its effectiveness and reliability.

References

Dataset of standard 512X512 Grayscale text images. Retrieved 2014, 2014, from http://decsai.ugr.es/cvg/ CG/base.htm.

Aiswarya, K., V. Jayaraj and D. Ebenezer. 2010. A new and efficient algorithm for the removal of high density salt and pepper noise in images and videos. In: Computer Modeling and Simulation, 2010. ICCMS'10. Second International Conference on, pp. 409-413.

Astola, J. and P. Kuosmanen 1997. Fundamentals of nonlinear digital filtering, CRC press pp. 
Esakkirajan, S., T. Veerakumar, A. N. Subramanyam and C. PremChand. 2011. Removal of high density salt and pepper noise through modified decision based unsymmetric trimmed median filter. Signal Processing Letters, IEEE 18: 287-290.

Hwang, H. and R. Haddad. 1995. Adaptive median filters: new algorithms and results. Image Processing, IEEE Transactions on 4: 499-502.

Jayaraj, V. and D. Ebenezer. 2010. A new switching-based median filtering scheme and algorithm for removal of high-density salt and pepper noise in images. EURASIP Journal on Advances in Signal Processing . http://asp.eurasijournals.com/context/1/690218

Junez-Ferreira, C. A., F. A. Velasco-Avalos and N. PastorGomez. A Salt and Pepper Noise Removal and Restoration Refinement Algorithm. www.iiis.org/.../ za059sf.pdf
Ng, P.-E. and K.-K. Ma. 2006. A switching median filter with boundary discriminative noise detection for extremely corrupted images. Image Processing, IEEE Transactions on 15: 1506-1516.

Srinivasan, K. and D. Ebenezer. 2007. A new fast and efficient decision-based algorithm for removal of highdensity impulse noises. Signal Processing Letters, IEEE 14: 189-192.

Wang, Z., A. C. Bovik, H. R. Sheikh and E. P. Simoncelli. 2004. Image quality assessment: from error visibility to structural similarity. Image Processing, IEEE Transactions on 13: 600-612.

Weber, A. 1997. The USC-SIPI image database. Signal and Image Processing Institute of the University of Southern California. URL: http://sipi. usc. edu/ services/database

Zhang, S. and M. A. Karim. 2002. A new impulse detector for switching median filters. Signal Processing Letters, IEEE 9: 360-363. 
Nepal Journal of Science and Technology Vol. 15, No.2 (2014) 123-132 